\title{
Early Detection of Parkinson Disease Progression using Gaussian Naïve Bayes Machine Learning Approach by identifying Degeneration in Basal Ganglia Regions
}

\author{
Madhusudhana G K, M B Sanjaypande, Raveesh B N
}

\begin{abstract}
Parkinson's Disease (PD) is a progressive neuro-degenerative disorder that affects millions of people across the globe. Analyzing volume changes in the basal ganglia seems to be a promising approach towards developing non-invasive and non-radioactive neuro-imaging markers for this disease. In this work, we report a study of classification based on the volumes of basal ganglia regions obtained from brain atlas. The study investigates the volume changes in certain anatomical structures of the basal ganglia region of PD affected subjects.
\end{abstract}

Keywords : basal ganglia, brain atlas, neuro-degenerative disorder, Parkinson's disease.

\section{INTRODUCTION}

$\mathrm{P}_{\mathrm{a}}$ arkinson's disease is a progressive neuro-degenerative disorder that affects millions of people across the globe [1]. Huge numbers of the side effects are because of lost neurons that produce a substance courier in the cerebrum called dopamine. At the point when dopamine levels decline, it causes unusual mind action, prompting manifestations of Parkinson's malady.

The malady advances as cells are demolished in specific pieces of the cerebrum stem, especially the bow formed cell mass known as the substantia nigra. Nerve cells in the substantia nigra convey filaments to tissue situated in the two sides of the cerebrum. There the cells discharge basic synapses that help control development and coordination.

Diagnosis of Parkinson's disease (PD) is difficult in the early premotor stages based on clinical evidence. Still no remarkable standard symptomatic test for PD is accessible,

Revised Manuscript Received on October 25, 2020.

* Correspondence Author

Madhusudhana G K*, Research Scholar, Department of Computer Science and Engineering, VVIET, Visvesvaraya Technological University, Belagavi, India. Email: madhugk06@gmail.com.

Dr. M B Sanjaypande, Research Supervisor, Department of Computer Science and Engineering, VVIET, Visvesvaraya Technological University, Belagavi, India. Email: rakroop99@gmail.com.

Dr. Raveesh B N, Professor and Head, Department of Psychiatry, Mysore Medical College, Mysore, India, Email: raveesh6@gmail.com.

(c) The Authors. Published by Blue Eyes Intelligence Engineering and Sciences Publication (BEIESP). This is an open access article under the CC BY-NC-ND license (http://creativecommons.org/licenses/by-nc-nd/4.0/) however the innovations to create precise biomarkers dependent on blood tests or imaging examines have been investigated. The most reliable diagnostic method for PD is to evaluate dopaminergic function in the basal ganglia or mid brain regions by using various PET or SPECT imaging. But these modalities make use of radioactive substances and can also be very expensive. Presently, Anatomic X-ray, a non obtrusive methodology, can't legitimately uncover dopaminergic neuron misfortune in PD [2] ]

Consequently, breaking down the related dissemination fiber volume changes in the basal ganglia is by all accounts a promising methodology towards creating non-intrusive and non-radioactive neuro-imaging markers for this disorder.

In this work, we report a study of classification based on the volumes of basal ganglia regions obtained from brain atlas. The study investigates the fibre volume changes in certain anatomical structures of the basal ganglia region of $\mathrm{PD}$ affected subjects.

\section{DATASET}

Despite the fact that it is critical to assess the Substantia Nigra in patients with Parkinsonian condition, it is hard to portray its life systems, even by MR imaging. Anatomic portrayal of the course of nerve strands around the Substantia Nigra from brain atlas is used to evaluate its volume changes in patients with Parkinson's disease. The diffusion-weighted MR images are used for this purpose.

fMRI scanned images have been taken from two datasets for this study. First dataset is from neorocon, National Institute for Research and Development in Informatics, Romania, populated from 43 subjects consisting of 27 patients and 16 normal controls[5]. The dataset contain T1 and resting-state scans with voxel size of $0.97 \times 0.97 \times 1 \mathrm{~mm}$.

\section{METHODOLODY}

\section{A. Defining regions of interest(ROI).}

The main focus in this study is investigating volume changes in the midbrain regions. For this purpose, a volume analysis was performed within the Substantia Nigra (left; right), striatum, SNc, SNr and Red neucleus. The volumes of all ROIs were derived using brain structural atlas of DSI studio. 


\section{Early Detection of Parkinson Disease Progression using Gaussian Naïve Bayes Machine Learning Approach by identifying Degeneration in Basal Ganglia Regions}

Basal Ganglia consists of Substantia Nigra (both left and right), Red neucleus, SNc, SNr and straitum. Sample snapshots of volumes of ROIs are shown in Figure-1.

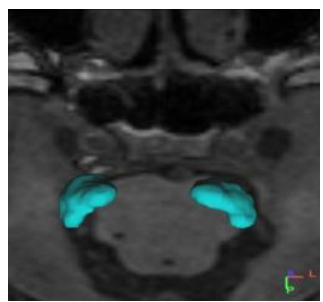

(a) Male normal

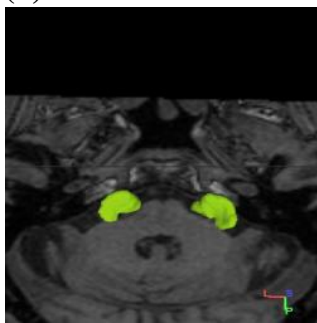

(c) Male PD

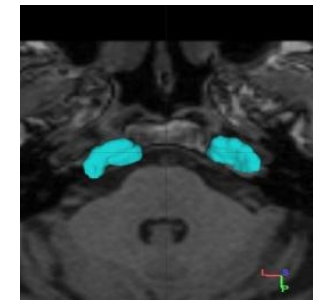

(b) Female normal

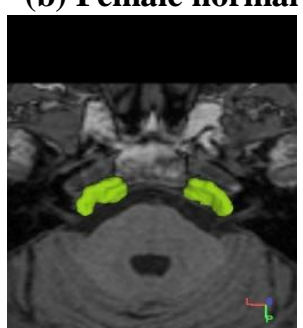

(d) Female PD
Fig.1. ROIs for normal and PD subjects

\section{B. Volume Measurement of ROIs}

The midbrain, which included the center Substantia Nigra (left; right), striatum , SNc, SNr and Red neucleus, of all 43 subjects were marked and $3 \mathrm{D}$ volume was obtained from the Atlas. The voxel counts and volumes of each structure were recorded by viewing the corresponding region report.

In the patients with Parkinson's disease $(n=20)$, the average volume of the substantia nigra for men was $249 \mathrm{~mm}$ and women was $196 \mathrm{~mm}$. Volume of substantia nigra and red neucleus were reduced in PD subjects as compared with normal subjects. Volume of striatum , SNc and SNr did not deviate much between PD and normal subjects. A gender based comparison of the volume of the substantia nigra, and Red nucleus between the control subjects and the patients with Parkinson's is shown in Table I and Table II.

Table- I: Average volume of ROIs

Table- II: Average volume of substantia Niagra (Left:Right)

\begin{tabular}{|c|c|c|c|c|}
\hline \multirow{4}{*}{ Gender } & \multicolumn{2}{|c|}{$\begin{array}{c}\text { Parameters in PD } \\
\text { patients }\end{array}$} & \multicolumn{2}{c|}{$\begin{array}{c}\text { Parameters in } \\
\text { NON-PD patients }\end{array}$} \\
\cline { 2 - 5 } & $\begin{array}{c}\text { Avg. } \\
\text { volume of } \\
\text { substantia } \\
\text { Nigra left }\end{array}$ & $\begin{array}{c}\text { Avg. } \\
\text { volume of } \\
\text { substantia } \\
\text { Nigra right }\end{array}$ & $\begin{array}{c}\text { Avg. } \\
\text { volume of } \\
\text { substantia } \\
\text { Nigra left }\end{array}$ & $\begin{array}{c}\text { Avg. } \\
\text { volume of } \\
\text { substantia } \\
\text { Nigra } \\
\text { right }\end{array}$ \\
\hline \multirow{2}{*}{ Male } & $\begin{array}{c}235.6 \\
\mathrm{~mm}^{3}\end{array}$ & $\begin{array}{c}227.13 \\
\mathrm{~mm}^{3}\end{array}$ & $\begin{array}{c}270.82 \\
\mathrm{~mm}^{3}\end{array}$ & $\begin{array}{c}259.87 \\
\mathrm{~mm}^{3}\end{array}$ \\
\hline Female & $\begin{array}{c}214.98 \\
\mathrm{~mm}^{3}\end{array}$ & $\begin{array}{c}206.65 \\
\mathrm{~mm}^{3}\end{array}$ & $\begin{array}{c}242.23 \\
\mathrm{~mm}^{3}\end{array}$ & $\begin{array}{c}237.46 \\
\mathrm{~mm}^{3}\end{array}$ \\
\hline
\end{tabular}

\section{CLASSIFICATION}

Since the data values are continuous, average suppositions that will be that the persistent qualities related with each class

\begin{tabular}{|c|c|c|c|c|}
\hline \multirow{4}{*}{ Gender } & \multicolumn{2}{|c|}{$\begin{array}{c}\text { Parameters in PD } \\
\text { patients }\end{array}$} & \multicolumn{2}{c|}{$\begin{array}{c}\text { Parameters in } \\
\text { NON-PD patients }\end{array}$} \\
\cline { 2 - 5 } & $\begin{array}{c}\text { Avg. } \\
\text { volume of } \\
\text { substantia } \\
\text { Nigra }\end{array}$ & $\begin{array}{c}\text { Avg. } \\
\text { volume } \\
\text { of Red } \\
\text { nucleus }\end{array}$ & $\begin{array}{c}\text { Avg. } \\
\text { volume of } \\
\text { substantia } \\
\text { Nigra }\end{array}$ & $\begin{array}{c}\text { Avg. } \\
\text { volume } \\
\text { of Red } \\
\text { nucleus }\end{array}$ \\
\hline \multirow{2}{*}{ Male } & 249.6 & 684 & 265 & 719 \\
& $\mathrm{~mm}$ & $\mathrm{~mm}$ & $\mathrm{~mm} 3$ & $\mathrm{~mm} 3$ \\
\hline \multirow{2}{*}{ Female } & 196 & 651 & 216 & 666 \\
& $\mathrm{~mm}$ & $\mathrm{~mm}$ & $\mathrm{~mm}$ & $\mathrm{~mm} 3$ \\
\hline
\end{tabular}

are conveyed by a typical (or Gaussian) distribution. Gaussian Naive Bayes algorithm [4] was used for classification since it fits the assumption of Gaussian distribution.

The preparing information contains persistent estimations of volumes of substantia nigra left and substantia nigra right (x). We first segment the information by the class, and afterward process the mean and difference of volume credits in each class ' $c$ ' ('0', normal, ' 1 ' PD). Let 'Mean' be the mean of the qualities in 'x' related with class 'c', and let be the change of the qualities in related with class 'c'. We have quality qualities for 'xi'. At that point, the likelihood distribution of the property estimations given a class 'c', $P(x i \mid c)$, can be computed by plugging values into the equation for a normal distribution parameterized by 'mean' and 'sigma ${ }^{2}$. That is,

$$
P(x i \mid c)=\frac{1}{\sqrt{2 \times \Pi \times \operatorname{sigma}_{x i, c}^{2}}} \times \exp \left(-\frac{\left(x_{i}-\text { mean }_{x i, c}\right)^{2}}{2 \times \operatorname{sigma}_{x i, c}^{2}}\right)
$$

At long last, the restrictive likelihood of each class given a case (test example) is determined utilizing Bayes Hypothesis as shown in equation(1).

Table- III: Accuracy of Naïve Bayes classifier

\begin{tabular}{|c|l|l|}
\hline $\begin{array}{c}\text { Number of } \\
\text { Training samples }\end{array}$ & $\begin{array}{c}\text { Number of Test } \\
\text { samples }\end{array}$ & Accuracy \\
\hline $25(60 \%)$ & $18(40 \%)$ & $77.77 \%$ \\
\hline $30(70 \%)$ & $13(30 \%)$ & $77.13 \%$ \\
\hline $36(80 \%)$ & $7(20)$ & $78.36 \%$ \\
\hline $34(95 \%)$ & $3(5 \%)$ & $79.41 \%$ \\
\hline
\end{tabular}

\section{RESULTS AND DISCUSSION}

In all non PD images, the substantia nigra appeared quite big with uniform surface area on the 3D atlas. Both substantia nigra left and substantia nigra right and also red nucleus were clearly noticeable in these images. Figure-1 depicts substantia nigra left and substantia nigra right in non PD and PD subjects for both male and female subjects.

The volumes of substatia nigra were less in male PD than Non-PD subjects irrespective of gender as shown in Table-I and Table-II. The average area of the red nucleus was 684 $\mathrm{mm} 3$ in the male PD group, $719 \mathrm{~mm}^{3}$ in the male non PD, $666 \mathrm{~mm}^{3}$ in the non PD female and $651 \mathrm{~mm} 3$ in the male PD. Even though, the volume of red nuclei is less is PD subjects as compared to Non-PD subjects, significant reduction is not observed in female subjects.

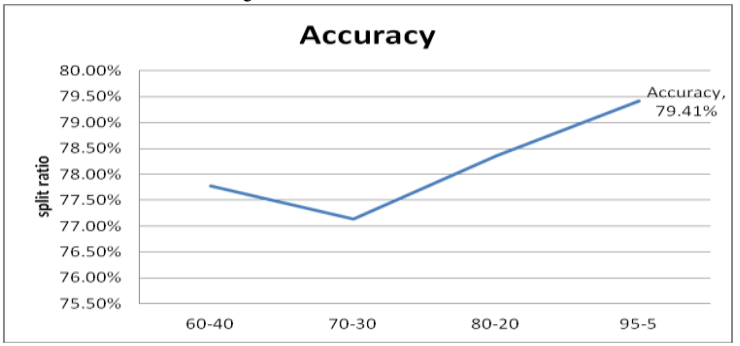

Fig 2: Accuracy of the classifier with change in split ratios.

Figure-2 depicts, a plot showing the accuracy of the classifier on various split ratios

Published By:

Blue Eyes Intelligence Engineering and Sciences Publication

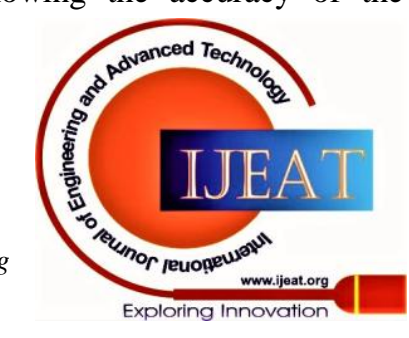


of training versus test samples. The classifier gives more accurate predictions as we increase the number of training samples.

The accuracy of the classifier also depends on the training dataset used. The volume difference in ROIs under consideration is less between PD and Non-PD subjects, since the dataset contains only early PD cases. Although other authors [3] claim to have found significant volume loss in the basal ganglia regions, we were not satisfied with the volume differences found. The volumes of the regions were dependent on the gender. The accuracy of the classifier can be increased if more data could be generated on moderate and severe PD cases, and significant volume differences could be captured.

\section{CONCLUSION}

Diffusion tensor images with atlas region rendering provide a convenient way to visualize degeneration of the basal ganglia regions viz substantia nigra left, substantia nigra right and red nucleus. Volume of substantia nigra and red neucleus are reduced in PD subjects as compared with normal subjects. The accuracy of the classifier is dependent on the training data and enhances as we increase the number of training samples. The results obtained encourage further studies for evaluating the correlation of degeneration in various basal ganglia regions with respect to age and gender.

\section{REFERENCES}

1. Timothy R. Mhyre, James T. Boyd, Robert W. Hamill, and Kathleen A. Maguire-Zeiss, "Parkinson's Disease", PMCID: PMC4372387, NIHMSID: NIHMS659855, PMID: 23225012, PMC 2015 March 24.

2. Gennaro Pagano, Flavia Niccolini, Marios Politis, "Imaging in Parkinson's disease", Clinical Medicine 2016 Vol 16, No 4: 371-5, Clinical Medicine 2016 Vol 16, No 4: 371-5.

3. Cyril Atkinson-Clement, Serge Pint, Alexandre Eusebi, Olivier Coulon, "Diffusion tensor imaging in Parkinson's disease: Review and meta-analysis", SciencecDirect, NeuroImage:Clinical 16, 2017.

4. Prateek Majumder. (2020, May 10). Gaussian Naive Bayes [Online]. Available: https://iq.opengenus.org/gaussian-naive-bayes/

5. Neurocon Image Repository, (2020, Jan 08) National Institute for Research and Development in Informatics, Romania, Department of Neurobiology, Beijing Institute of Geriatrics, Xuanwu Hospital, Capital Medical University, Parkinson Disease Centre of Beijing Institute for Brain Disorders.China,

6. [Online]Available:

http://fcon_1000.projects.nitrc.org/indi/retro/parkinsons.html

\section{AUTHORS PROFILE}

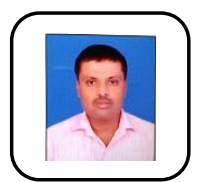

Madhusudhana G K. Assistant Professor at Vidya Vikas Institute of Engineering and Technology, Mysore. Pursuing $\mathrm{PhD}$ from Visvesvaraya Technological University, Belagavi.

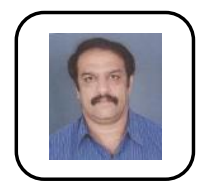

Dr. M B Sanjaypande. Professor at GMIT, Davangere. Obtained his PhD from University of Mysore,

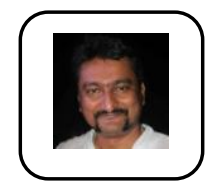

Dr. Raveesh B N, Professor and Head, Department of Psychiatry, Mysore Medical College, Mysore.

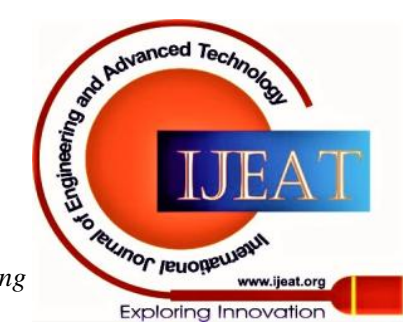

